Research Article

\title{
Incorporation of Structural Health Monitoring Coupled Data in Dynamic Extreme Stress Prediction of Steel Bridges Using Dynamic Coupled Linear Models
}

\author{
Xueping Fan (iD, Zhipeng Shang, Guanghong Yang, Xiaoxiong Zhao, and Yuefei Liu \\ School of Civil Engineering and Mechanics, Lanzhou University, Lanzhou 730000, China \\ Correspondence should be addressed to Xueping Fan; fanxp@lzu.edu.cn
}

Received 15 January 2020; Revised 4 June 2020; Accepted 24 July 2020; Published 11 August 2020

Academic Editor: Abdul Aziz bin Abdul Samad

Copyright (c) 2020 Xueping Fan et al. This is an open access article distributed under the Creative Commons Attribution License, which permits unrestricted use, distribution, and reproduction in any medium, provided the original work is properly cited.

\begin{abstract}
In this article, an approach for using structural health monitoring coupled extreme stress data in dynamic extreme stress prediction of steel bridges is presented, where the coupled extreme stress data means the extreme stress data with dynamicity, randomness, and trend. Firstly, the modeling processes about dynamic coupled linear models (DCLM) are provided based on a supposed coupled time series; furthermore, the dynamic probabilistic recursion processes about DCLM are given with Bayes method; secondly, the monitoring dynamic coupled extreme stress data is taken as a time series, historical monitoring coupled extreme stress data-based DCLM and the corresponding Bayesian probabilistic recursion processes are given for predicting bridge extreme stresses; furthermore, the monitoring mechanism is provided for monitoring the prediction precision of DCLM; finally, the monitoring coupled extreme stress data of a steel bridge is used to illustrate the proposed approach which can provide the foundations for bridge reliability prediction and assessment.
\end{abstract}

\section{Introduction}

Structural health monitoring (SHM) is a promising technology to improve the safety of civil infrastructures and achieve their sustainable management. For steel bridges, structural safety is very important, and the monitoring extreme stress data provided by SHM systems is an important parameter for structural safety analysis and can be used for evaluating and predicting structural dynamic safety reliability.

During long-term service periods, many steel bridges are subjected to many random dynamic loads like vehicle load, temperature load, and wind load; even these loads occur simultaneously. Therefore, the accumulated extreme stress data by structural health monitoring (SHM) system commonly has the characteristic of coupling including randomness, dynamicity, and tendency [1]; furthermore, based on the monitoring coupled extreme stress data which is considered as Markov chains, dynamic coupled extreme stress prediction for steel bridges is particularly important as bridges age, which could reflect bridge safety and help to guide the important decisions regarding the structural preventive maintenance. Therefore, monitoring coupled data-based extreme stress prediction and assessment of steel bridges is accordingly a grand challenge of SHM field $[2,3]$.

In recent years, SHM has become the escalating urgent need for modern bridge engineering and grew into a hot topic on both investments and researches around the world. With the innovation of sensing data acquisition, SHM systems are comprehensively deployed and used for obtaining the coupled extreme stress data of steel bridges in different sampling frequency. How to make reasonable use of these data for predicting structural extreme stresses has been one of the main scientific problems in the SHM field. So far, the researches on SHM have generally experienced two stages. The first stage, falling in the mature stage, is to install an array of sensors for monitoring, collection, and recovery of SHM data during the long-term service periods [4-12]. The second stage is mainly the application of SHM data. Most studies are mainly focused on the modal parameter 
identification $[13,14]$, structural damage detection technology $[10,13,15,16]$, model updating [3], performance prediction [17-19], and reliability assessment [20-22]. The second stage shows that some researches about the application of SHM data have been conducted, but, in the analysis processes, the coupling of SHM data is not reasonably taken into account. Therefore, monitoring coupled data-based dynamic prediction of structural coupled extreme stresses remains at the initial research stage. At present, although some achievements about structural response prediction have been made, most of them have some limitations; for example, dynamic linear models and Gaussian mixed particle filter-based extreme stress prediction method of steel bridges are proposed, but, in the analysis processes, the coupling of monitoring extreme stress data is not reasonably considered [4]; a data assimilation method about Bayesian Fourier dynamic linear prediction of periodic extreme stresses for steel bridges is proposed; in the analysis processes, the modeling processes of dynamic linear models are complex and are not generally used [23]; the ARMA models [24] suitable for short-term prediction and BDLM [17, 18] without steady prediction precision are not clearly satisfying for predicting coupled performance information; the performance prediction method through decoupling the monitoring coupled data decreases the coupling effect on the prediction results to a certain extent, but the decoupling itself is a difficult problem due to the complexity of the coupled information, and it can lead to information losses and so does its work in the prediction processes [25]; the traditional nonlinear filtering methods are usually used to build the prediction models based on curve fitting of historical dynamic data [26], so they are very subjective, and monitoring load effects tend to exhibit the coupling; thus, the traditional subjective nonlinear filtering methods will affect the precision and accuracy of the prediction results; the common regression models derived from the historical monitoring data are also very subjective and are not considering the uncertainty about the monitoring data $[27,28]$; the support vector regression model needs a lot of monitoring data to be trained for completing the model prediction, which consumes a lot of time [29]. In view of the above limitations, a Bayesian dynamic coupled linear prediction method of the coupled extreme stresses for steel bridges could be explored and is the topic of the present research.

Based on the above issues, this paper proposes the Bayesian dynamic coupled linear prediction method of coupled extreme stresses for steel bridges based on the monitoring coupled extreme stress data. Firstly, based on a supposed coupled time series, dynamic coupled linear models (DCLM) are built, and the corresponding dynamic probabilistic recursion processes are proposed with Bayes method (see Section 2); secondly, based on Section 2, monitoring coupled extreme stress data-based DCLM and the corresponding Bayesian dynamic probabilistic recursion processes are provided for predicting coupled extreme stresses in Section 3; furthermore, the monitoring mechanism about DCLM is provided for monitoring the abnormal coupled extreme stress data (see Section 4). Finally, the effectiveness of the proposed DCLM is compared with several existing methods through the monitoring coupled extreme stress data from a steel bridge in Section 5, and some conclusions are drawn in Section 6. The main flowcharts of this paper are shown in Figure 1.

\section{The General Coupled Time Series-Based Dynamic Coupled Linear Models (DCLM) and the Corresponding Bayesian Probabilistic Recursion}

The flowcharts of Section 2 are shown in Figure 2.

According to Figure 2, the detailed contents of Section 2 are described as follows.

2.1. DCLM Based on a General Coupled Time Series. DCLM include state equation, monitored equation, and the initial coupled state information, where the state equation shows changes of the system with time and reflects inner dynamic changes of the system and random disturbances, the monitored equation expresses the relationship between the monitored data and the current state parameters of the system, and the initial coupled state information is expressed with the mixture of a few normal probability density functions (PDFs) about the initial coupled state. In this chapter, the general coupled time series is adopted to build DCLM, and the detailed modeling methods are described as follows.

It is supposed that there is a general dynamic coupled time series set $\mathbf{D}_{t}$ with $(n-1)$-order local trends, which includes the data at and before time $t$. The state set, also taken as a coupled time series approximately obtained with cubical smoothing algorithm with five-point approximation $[1,2,17]$ through resampling $\mathbf{D}_{t}$, is denoted with $\mathbf{D}_{\theta_{t}}$, where $\theta_{t}$ is the state at time $t, \mathbf{D}_{\theta_{t}}$ includes all the state data at and before time $t$, and the $i^{\text {th }}$ difference data set of $\mathbf{D}_{\theta_{t}}$ is $\mathbf{D}_{\theta_{t, i}}$. In general, for the general coupled time series set $\mathbf{D}_{t}$ with $(n-1)$-order local trends, difference can be carried out until the time series after difference is stationary, and the number $(n-1)$ of difference is the order of local trends. Besides, the Augmented Dickey-Fuller (ADF) test method [30-33] can also help ascertain the stationarity of the data before and after difference. Namely, ADF test method can help determine the order of local trends.

Let $\left\{\theta_{t}, t \in\{1,2, \ldots\}\right\}$ be the state time series; there are the following difference equations:

$$
\begin{aligned}
\Delta^{0} \theta_{k}= & \theta_{k}, \\
\Delta^{1} \theta_{k}= & \theta_{k+1}-\theta_{k}, \\
\Delta^{2} \theta_{k}= & \Delta^{1} \theta_{k+1}-\Delta^{1} \theta_{k}, \\
& \cdots, \\
\Delta^{i} \theta_{k}= & \Delta^{i-1} \theta_{k+1}-\Delta^{i-1} \theta_{k}, \\
& \cdots, \\
\Delta^{n-1} \theta_{k}= & \Delta^{n-2} \theta_{k+1}-\Delta^{n-2} \theta_{k},
\end{aligned}
$$

where $\left\{\Delta^{i} \theta_{k}, i \in\{1,2, \ldots, n-1\}, k \in\{1,2, \ldots, t\}\right\}$ is the $i^{\text {th }}$ order difference data about $\theta_{k}$. 


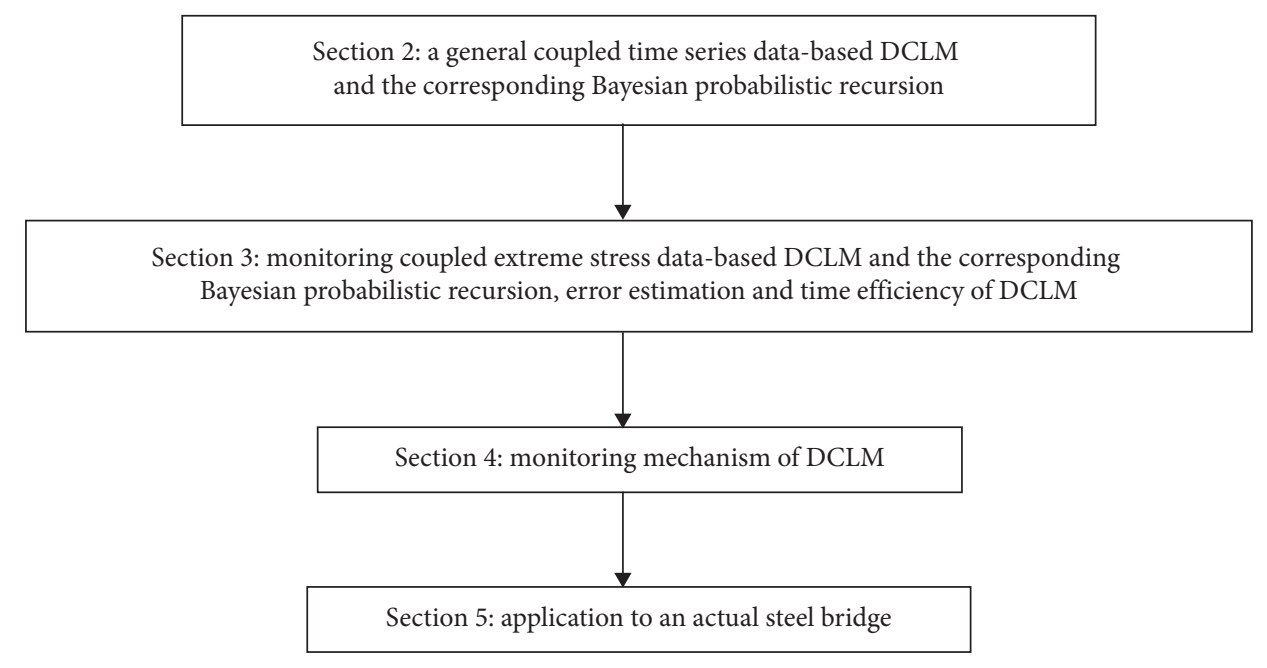

Figure 1: Main flowcharts of this paper.

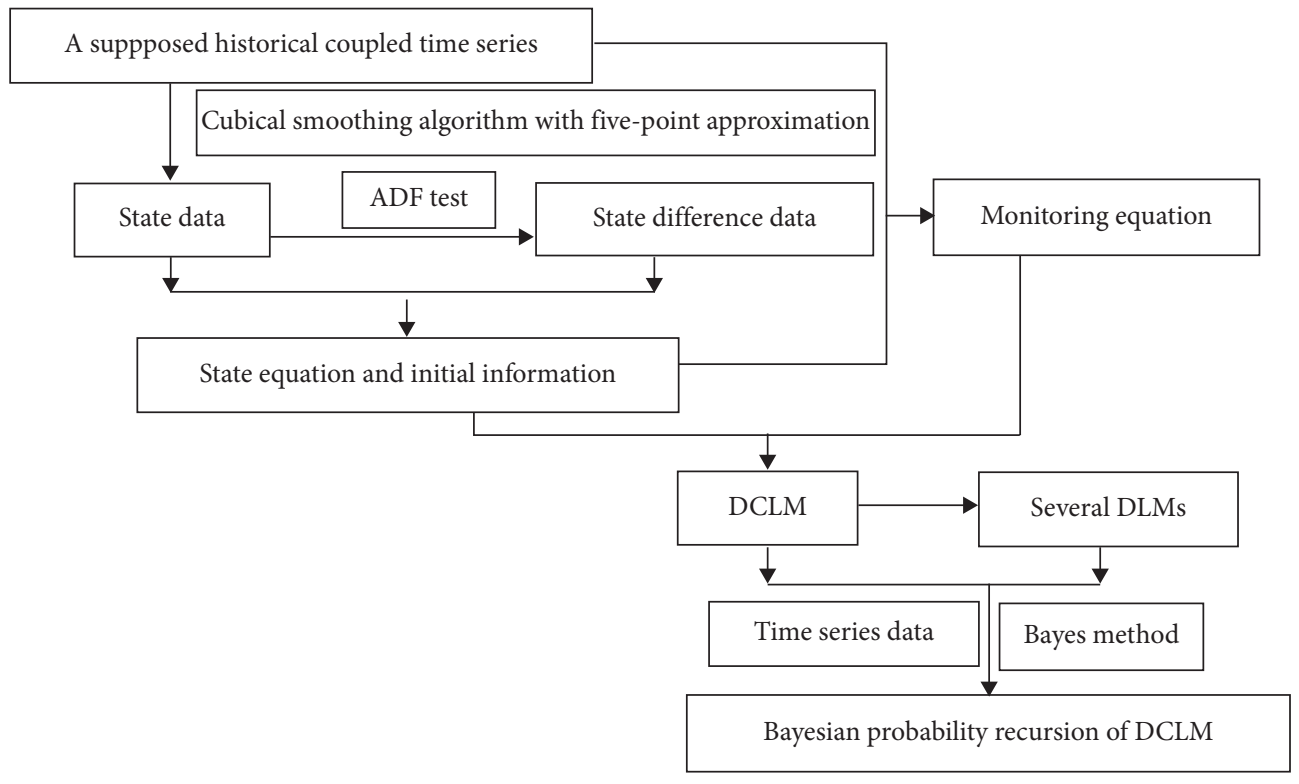

Figure 2: The flowcharts of Section 2.

Furthermore, (2) can be obtained as

$$
\begin{gathered}
\theta_{k+1}=\theta_{k}+\Delta^{1} \theta_{k}, \\
\Delta^{1} \theta_{k+1}=\Delta^{1} \theta_{k}+\Delta^{2} \theta_{k}, \\
\cdots, \\
\Delta^{i-1} \theta_{k+1}=\Delta^{i-1} \theta_{k}+\Delta^{i} \theta_{k}, \\
\cdots, \\
\Delta^{n-2} \theta_{k+1}=\Delta^{n-2} \theta_{k}+\Delta^{n-1} \theta_{k} .
\end{gathered}
$$

Suppose that all the dynamic state variables $\left\{\theta_{t, i}, i \in\{1,2, \ldots, n\}\right\}$ are all Markov chains and are internally independent and mutually independent between each other. Taking into account the monitoring error $v_{t}$ about $y_{t}$ conditional on the state (or level) $\theta_{t, 1}$ and transition error $w_{t, j}$ from state $\theta_{t-1, j}+\theta_{t-1, j+1}$ to $\theta_{t, j}$, the $(n-1)$-order DCLM are defined in the following form.

Monitoring equation is

$$
\begin{aligned}
y_{t} & =\theta_{t, 1}+v_{t}, v_{t} \sim \mathrm{N}\left(0, e^{-0.0004 t^{2}} V\right) \\
\Rightarrow y_{t} & =\mathbf{F} \boldsymbol{\theta}_{t}+v_{t}, v_{t} \sim \mathrm{N}\left(0, V_{t}\right), \\
\mathbf{F} & =\left(\begin{array}{lllll}
1 & 0 & 0 & \ldots & 0
\end{array}\right)_{1 \times n}, \\
\theta_{t} & =\left(\begin{array}{lllll}
\theta_{t, 1} & \theta_{t, 2} & \theta_{t, 3} & \ldots & \theta_{t, n}
\end{array}\right)_{1 \times n}^{T} .
\end{aligned}
$$

State equation is 


$$
\begin{aligned}
& \theta_{t, j}=\theta_{t-1, j}+\theta_{t-1, j+1}+w_{t, j}, w_{t, j} \sim \mathrm{N}\left(0, W_{t, j}\right), \\
& j \in\{1,2, \ldots, n-1\}, \\
& \theta_{t, n}=\theta_{t-1, n}+w_{t, n}, w_{t, n} \sim \mathrm{N}\left(0, W_{t, n}\right), \\
& \Rightarrow \boldsymbol{\theta}_{t}=\mathbf{G} \boldsymbol{\theta}_{t-1}+\mathbf{w}_{t}, \mathbf{w}_{t} \sim \mathrm{N}\left(0, \mathbf{W}_{t}\right), \\
& \boldsymbol{\theta}_{t}=\left(\begin{array}{lllll}
\theta_{t, 1} & \theta_{t, 2} & \theta_{t, 3} & \ldots & \theta_{t, n}
\end{array}\right)_{1 \times n}^{T}, \\
& \mathbf{w}_{t}=\left(\begin{array}{lllll}
w_{t, 1} & w_{t, 2} & w_{t, 3} & \ldots & w_{t, n}
\end{array}\right)_{1 \times n}^{T},
\end{aligned}
$$

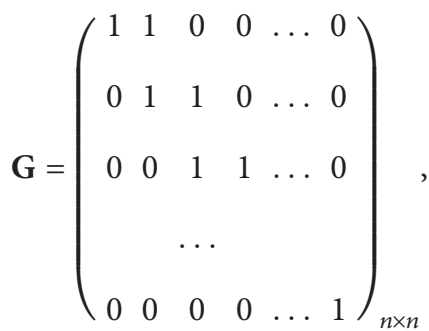

$$
\begin{aligned}
& \mathbf{W}_{t}=\left(\begin{array}{ccccc}
W_{t, 1} & 0 & 0 & \ldots & 0 \\
0 & W_{t, 2} & 0 & \ldots & 0 \\
0 & 0 & W_{t, j} & \ldots & 0 \\
& & \ldots & & \\
0 & 0 & 0 & 0 & W_{t, n}
\end{array}\right)_{n \times n} .
\end{aligned}
$$

Through the least residual error quadratic sum method [2], initial coupled state information can be obtained with

$$
\begin{aligned}
& p\left(\theta_{t-1,1} \mid \mathbf{D}_{\theta_{t-1,1}}\right) \approx \sum_{i_{1}=1}^{G_{1}} \alpha_{t-1}^{i_{1}} \mathrm{~N}\left(\theta_{t-1,1} ; \mu_{t-1}^{i_{1}}, \phi_{t-1}^{i_{1}}\right) ; \\
& p\left(\theta_{t-1,2} \mid \mathbf{D}_{\theta_{t-1,2}}\right) \approx \sum_{i_{2}=1}^{G_{2}} \alpha_{t-1}^{i_{2}} \mathrm{~N}\left(\theta_{t-1,2} ; \mu_{t-1}^{i_{2}}, \phi_{t-1}^{i_{2}}\right), \\
& \ldots \\
& p\left(\theta_{t-1, i} \mid \mathbf{D}_{\theta_{t-1, i}}\right) \approx \sum_{i_{\beta}=1}^{G_{i}} \alpha_{t-1}^{i_{\beta}} \mathrm{N}\left(\theta_{t-1, i} ; \mu_{t-1}^{i_{\beta}}, \phi_{t-1}^{i_{\beta}}\right) ; \\
& \ldots \\
& p\left(\theta_{t-1, n} \mid \mathbf{D}_{\theta_{t-1, n}}\right) \approx \sum_{i_{n}=1}^{G_{n}} \alpha_{t-1}^{i_{n}} \mathrm{~N}\left(\theta_{t-1, n} ; \mu_{t-1}^{i_{n}}, \phi_{t-1}^{i_{n}}\right),
\end{aligned}
$$

where $y_{t}$ is the data at time $t ; v_{t}$ is the monitoring error; $\mathrm{N}(\bullet)$ is normal probability density function (PDF); $V$ is the initial error variance obtained with the historical time series data; $\theta_{t, i}$ is the data at time $t$ from $\mathbf{D}_{\theta_{t, i}} ; \theta_{t, 1}$ is the state (or level) at time $t$, which between times $t-1$ and $t$ changes by the addition of $\theta_{t-1,2}$ plus the noise $w_{t, 1} ; \theta_{t-1,2}$ represents a systematic change in state (or level), which itself changes by the addition of $\theta_{t-2,3}$ plus noise; proceeding through the state parameters for $j \in\{1,2, \ldots, n-1\}$, each $\theta_{t, j}$ changes systematically via the increment $\theta_{t-1, j+1}$ and also by the addition of the noise term $w_{t, j}$; the $n^{\text {th }}$ component $\theta_{t, n}$ changes only stochastically; $T$ is the symbol of transpose; $\mathbf{w}_{t}=\left(w_{t, 1}, \ldots, w_{t, n}\right)^{T}$ is the state error vector and $\mathbf{w}_{t} \sim \mathrm{N}\left(0, \mathbf{W}_{t}\right)$, where $\mathbf{W}_{t}$ is the variance matrix; $v_{t}$ and $\mathbf{w}_{t}$ are internally independent and mutually independent between each other. $\left\{G_{i}, i \in\{1,2, \ldots, n\}\right\}$ denotes the total number of normal PDFs about mixed Gaussian PDF, $\alpha_{t-1}^{i_{\beta}}$ is the $i_{\beta}^{\text {th }}$ mixed weight, and $\mathrm{N}\left(\theta_{t-1, i} ; \mu_{t-1}^{i_{\beta}}, \phi_{t-1}^{i_{\beta}}\right)$ is a Gaussian PDF with the mean $\mu_{t-1}^{i_{\beta}}$ and the variance $\phi_{t-1}^{\hat{\beta}_{\beta}}$.

2.2. Decomposition Model (Dynamic Linear Model (DLM)) of DCLM. Due to the coupling of a general time series, initial coupled state information shown in (5) includes multiple mixture Gaussian PDFs; thus, there are $\prod_{i=1}^{n} G_{i}$ DLMs that can be built and used to make dynamic probabilistic recursion of the DCLM. Each dynamic linear model (DLM) can be, respectively, obtained with equations (3), (4), and (6).

Monitoring equation about the $J^{\text {th }}$ DLM is the same as (3), where $V_{t}$ is replaced by $V_{t, J}$.

State equation about the $J^{\text {th }}$ DLM is the same as (4), where $\mathbf{W}_{t}$ is replaced by $\mathbf{W}_{t, \mathrm{~J}}$.

Initial information about the $J^{\text {th }}$ DLM is

$$
\begin{aligned}
& p\left(\theta_{t-1,1} \mid \mathbf{D}_{\theta_{t-1,1}}\right) \approx \mathrm{N}\left(\theta_{t-1,1} ; \mu_{t-1}^{i_{1}}, \phi_{t-1}^{i_{1}}\right), \quad i_{1} \in\left\{1,2, \ldots, G_{1}\right\} \text {; } \\
& p\left(\theta_{t-1,2} \mid \mathbf{D}_{\theta_{t-1,2}}\right) \approx \mathrm{N}\left(\theta_{t-1,2} ; \mu_{t-1}^{i_{2}}, \phi_{t-1}^{i_{2}}\right), \quad i_{2} \in\left\{1,2, \ldots, G_{2}\right\} ; \\
& p\left(\theta_{t-1, i} \mid \mathbf{D}_{\theta_{t-1, i}}\right) \approx \mathrm{N}\left(\theta_{t-1, i} ; \mu_{t-1}^{i_{\beta}}, \phi_{t-1}^{i_{\beta}}\right), \quad i_{\beta} \in\left\{1,2, \ldots, G_{i}\right\} \\
& \text {...; } \\
& p\left(\theta_{t-1, n} \mid \mathbf{D}_{\theta_{t-1, n}}\right) \approx \mathrm{N}\left(\theta_{t-1, n} ; \mu_{t-1}^{i_{n}}, \phi_{t-1}^{i_{n}}\right), \quad i_{n} \in\left\{1,2, \ldots, G_{n}\right\}, \\
& \Rightarrow\left(\boldsymbol{\theta}_{t-1, J} \mid \mathbf{D}_{\theta_{t-1, J}}\right) \sim \mathrm{N}\left(\boldsymbol{\theta}_{t-1, J} ; \mathbf{M}_{t-1, J}, \mathbf{C}_{t-1, J}\right), \\
& J \in\left\{1,2, \ldots, \prod_{i=1}^{n} G_{i}\right\}, \\
& \boldsymbol{\theta}_{t-1, J}=\left(\begin{array}{lllll}
\theta_{t-1,1} & \theta_{t-1,2} & \theta_{t-1,3} & \ldots & \theta_{t-1, n}
\end{array}\right)_{1 \times n, J}^{T} ; \\
& \mathbf{D}_{\theta_{t-1, j}}=\left(\begin{array}{llllll}
\mathbf{D}_{\theta_{t-1,1}} & \mathbf{D}_{\theta_{t-1,2}} & \mathbf{D}_{\theta_{t-1,3}} & \ldots & \mathbf{D}_{\theta_{t-1, n}}
\end{array}\right)_{1 \times n, J}^{T} \\
& \mathbf{M}_{t-1, J}=\left(\begin{array}{lllll}
\mu_{t-1}^{i_{1}} & \mu_{t-1}^{i_{2}} & \mu_{t-1}^{i_{3}} & \ldots & \mu_{t-1}^{i_{n}}
\end{array}\right)_{1 \times n, J}^{T} ; \\
& \mathbf{C}_{t-1, J}=\left(\begin{array}{cccccc}
\phi_{t-1}^{i_{1}} & 0 & 0 & 0 & \ldots & 0 \\
0 & \phi_{t-1}^{i_{2}} & 0 & 0 & \ldots & 0 \\
0 & 0 & \phi_{t-1}^{i_{3}} & 0 & \ldots & 0 \\
& & & \ldots & & \\
0 & 0 & 0 & 0 & \ldots & \phi_{t-1}^{i_{n}}
\end{array}\right) \text {, }
\end{aligned}
$$

where $\mathbf{W}_{t, J}$ can be approximately solved with

$$
\mathbf{W}_{t, J}=-\mathbf{G C}_{t-1, J} \mathbf{G}^{T}+\frac{\mathbf{C}_{t-1, J}}{\delta},
$$

where $\mathbf{G}^{T}$ is the transpose of $\mathbf{G}$ and $\delta$ is the discount factor that is usually $0.48-0.98[2,17]$. 
2.3. Bayesian Probabilistic Recursion of DLM and DCLM Based on a General Coupled Time Series. Bayesian probabilistic recursion of DCLM means that dynamic recursive processes about DCLM are achieved with Bayes method $[34,35]$. Bayesian probabilistic recursion is good at inferring and predicting past and future parameters [34, 35], and Bayesian probabilistic recursive processes about DLM and DCLM are, respectively, shown as follows.

\subsubsection{Bayesian Probabilistic Recursion of DLM}

(1) With (4) and (6), the prior PDF about the state $\theta_{t, J}$ at time $t$ can be obtained with

$$
\left(\theta_{t, J} \mid \mathbf{D}_{\boldsymbol{\theta}_{t-1, J}}\right) \sim \mathrm{N}\left(\mathbf{a}_{t, J}, \mathbf{R}_{t, J}\right), \quad J \in\left\{1,2, \ldots, \prod_{i=1}^{n} G_{i}\right\},
$$

where $\mathbf{a}_{t, J}=\mathbf{G M}_{t-1, J}, \mathbf{R}_{t, J}=\mathbf{G C}_{t-1, J} \mathbf{G}^{T}+\mathbf{W}_{t, J}$.

(2) With (3) and (8), the one-step forward prediction PDF at time $t$ can be solved with

$$
\left(y_{t, J} \mid \mathbf{D}_{\theta_{t-1, J}}\right) \sim \mathrm{N}\left(f_{t, J}, Q_{t, J}\right), \quad J \in\left\{1,2, \ldots, \prod_{i=1}^{n} G_{i}\right\},
$$

where $f_{t, J}=\mathbf{F} \mathbf{a}_{t, J}$ can be used to predict the dynamic data of the $J^{\text {th }}$ DLM; $Q_{t, J}=\mathbf{F R}_{t, J} \mathbf{F}^{\mathrm{T}}+e^{-0.0004 t^{2}} V$. According to the definition of highest posterior density (HPD) region [34], the predicted interval of the $J^{\text {th }}$ DLM with a 95\% confidential interval at time $t$ is $\left[f_{t, J}-1.645 \sqrt{Q_{t, J}}, f_{t, J}+1.645 \sqrt{Q_{t, J}}\right]$.

(3) With Bayes method $[33,34],(8)$ and (9), while $y_{t, J}=$ $y_{t}$ is obtained, the posterior PDF about the state at time $t$ can be computed with

$$
\left(\boldsymbol{\theta}_{t, J} \mid \mathbf{D}_{\boldsymbol{\theta}_{t, J}}\right) \sim \mathrm{N}\left(\mathbf{M}_{t, J}, C_{t, J}\right), \quad J \in\left\{1,2, \ldots, \prod_{i=1}^{n} G_{i}\right\},
$$

where $\mathbf{M}_{t, J}=\mathbf{a}_{t, J}+\mathbf{A}_{\mathrm{t}, \mathrm{J}} e_{\mathrm{t}, \mathrm{J}}, \mathbf{C}_{t, J}=\mathbf{R}_{t, J}-\mathbf{A}_{\mathrm{t}, J} Q_{t, J} \mathbf{A}_{t, J}^{\mathrm{T}}, \mathbf{A}_{\mathrm{t}, J}=$ $\mathbf{R}_{t, J} \mathbf{F}^{\mathrm{T}} Q_{t, J}^{-1}, e_{t, J}=y_{t, J}-f_{t, J}, e_{t, J}$ is the one-step forward predicted error, and $y_{t, J}$ is the monitoring data at time $t . Q_{t, J}^{-1}$, the reciprocal of the one-step predicted variance, is the prediction precision of the $J^{\text {th }}$ DLM.

With (3)-(4) and (6)-(10), the Bayesian cyclic probabilistic recursive processes of the $J^{\text {th }}$ DLM can be achieved.

2.3.2. Bayesian Probabilistic Recursion of DCLM. With (3)-(10) and the distantly provided coupled data, Bayesian probabilistic recursive processes of DCLM with time-variant updated weighted coefficients are derived as follows.

(1) The a posteriori PDF about the state $\theta_{t-1}$ at time $t-1$ is

$$
\left(\boldsymbol{\theta}_{t-1} \mid \mathbf{D}_{\boldsymbol{\theta}_{t-1}}\right) \sim \sum_{J=1}^{\prod_{i=1}^{n} G_{i}} p_{t-1, J} \mathrm{~N}\left(\mathbf{M}_{t-1, J}, \mathbf{C}_{t-1, J}\right),
$$

where $\quad p_{t-1, J}=\left(\alpha_{t-1}^{i_{1, J}} \times \alpha_{t-1}^{i_{2, J}} \times \alpha_{t-1}^{i_{3, J}} \times \cdots \times \alpha_{t-1}^{i_{n, J}} /\right.$ $\left.\sum_{J=1}^{\prod_{i=1}^{n} G_{i}}\left(\alpha_{t-1}^{i_{1, J}} \times \alpha_{t-1}^{i_{2, J}} \times \alpha_{t-1}^{i_{3, J}} \times \cdots \times \alpha_{t-1}^{i_{n, J}}\right)\right)$ can be determined with (5) and (6).

(2) The prior PDF about the state $\theta_{t}$ at time $t$ is

$$
\left(\boldsymbol{\theta}_{t} \mid \mathbf{D}_{\boldsymbol{\theta}_{t-1}}\right) \sim \sum_{J=1}^{\prod_{i=1}^{n} G_{i}} p_{t-1, J} \mathrm{~N}\left(\mathbf{a}_{t, J}, \mathbf{R}_{t, J}\right)
$$

(3) The one-step prediction PDF at time $t$ is

$$
\left(y_{t} \mid \mathbf{D}_{\boldsymbol{\theta}_{t-1}}\right) \sim \sum_{J=1}^{\Pi_{i=1}^{n} G_{i}} p_{t-1, J} \mathrm{~N}\left(f_{t, J}, Q_{t, J}\right)
$$

where the predicted data, $f_{t}=\sum_{J=1}^{\prod_{i=1}^{n} G_{i}}$ $\left(p_{t-1, J} f_{t, J}\right),\left(\int_{-\infty}^{+\infty}\left(y_{t}-f_{t}\right)^{2} \sum_{J=1}^{\prod_{i=1}^{n} G_{i}} p_{t-1, J} \mathrm{~N}\left(f_{t, J}\right.\right.$, $\left.\left.Q_{t, J}\right) \mathrm{d} y_{t}\right)^{-1}$, is the prediction precision of DCLM. According to the definition of highest posterior density (HPD) region [34, 35], the predicted interval of the combinatorial DCLM with a $95 \%$ confidential

interval at time $t$ is $\left[\sum_{J=1}^{\prod_{i=1}^{n} G_{i}}\left(p_{t-1, J} f_{t, J}\right)-1.645\right.$

$\sqrt{\int_{-\infty}^{+\infty}\left(y_{t}-f_{t}\right)^{2}\left(\sum_{J=1}^{\prod_{i=1}^{n} G_{i}} p_{t-1, J} \mathrm{~N}\left(f_{t, J}, Q_{t, J}\right)\right) \mathrm{d} y_{t}}$,

$\sum_{J=1}^{\Pi_{i=1}^{n} G_{i}}\left(p_{t-1, J} f_{t, J}\right)+1.645$

$\left.\sqrt{\int_{-\infty}^{+\infty}\left(y_{t}-f_{t}\right)^{2}\left(\sum_{J=1}^{\Pi_{i=1}^{n} G_{i}} p_{t-1, J} \mathrm{~N}\left(f_{t, J}, Q_{t, J}\right)\right) \mathrm{d} y_{t}}\right]$.

(4) With Bayes method $[34,35]$, while $y_{t}$ is obtained, the posterior PDF about the state at time $t$ can be computed with

$$
\left(\boldsymbol{\theta}_{t} \mid \mathbf{D}_{\boldsymbol{\theta}_{t}}\right) \sim \sum_{J=1}^{\Pi_{i=1}^{n} G_{i}} p_{t, J} \mathrm{~N}\left(\mathbf{M}_{t, J}, \mathbf{C}_{t, J}\right),
$$

$$
\begin{aligned}
& \text { where } \quad p_{t, J}=\left(p_{t-1, J} p\left(y_{t, J} \mid \quad \mathbf{D}_{\theta_{t-1, J}}\right) / \sum_{J=1}^{\Pi_{i=1}^{n} G_{i}}\right. \\
& \left.\left(p_{t-1, J} p\left(y_{t, J} \mid \mathbf{D}_{\theta_{t-1, J}}\right)\right)\right)=\left(p_{t-1, J} \mathrm{~N}\left(f_{t, J}, Q_{t, J}\right) / \quad \sum_{J=1}^{\prod_{i=1}^{n} G_{i}}\right. \\
& \left.\left(p_{t-1, J} \mathrm{~N}\left(f_{t, J}, Q_{t, J}\right)\right)\right) \text {. }
\end{aligned}
$$

\subsection{Error Estimation and Time Efficiency Analysis of DCLM}

2.4.1. Error Estimation of DCLM. In this paper, mean square error (MSE) is adopted to measure the prediction errors, the MSE is smaller, and the corresponding model is better. The formula about MSE is 


$$
f_{\mathrm{MSE}}=\sqrt{\sum_{t=1}^{N} \frac{\left(f_{t}-y_{t}\right)^{2}}{\mathrm{~N}}}
$$

where $f_{\text {MSE }}$ is the formula of MSE; $N$ is the total number of the predicted data; $f_{t}$ is predicted with (13); $y_{t}$ is the monitoring data at time $t$. Some relations between MSE and sampling time intervals will be discussed in Sections 2.3.2 and 2.3.3.

2.4.2. Time Efficiency Analysis of DCLM. In order to test the time efficiency of DCLM, predicted coupled extreme stresses from different time intervals are considered for analysis comparison, and their MSEs are analyzed to discover different time efficiency. Hence, the every-hour, every-two-hours, and every-four-hours extreme stresses are predicted and utilized for illustrating the time efficiency of DCLM. The analysis results about the time efficiency of DCLM can be shown in Section 5.

\section{Monitoring Coupled Extreme Stress Data- Based DCLM and the Corresponding Bayesian Probabilistic Recursion}

Based on Figure 2, the flowcharts of monitoring coupled extreme stress data-based DCLM and the corresponding Bayesian probabilistic recursion can be obtained and shown in Figure 3.

According to Figure 3, the detailed analysis processes about monitoring coupled extreme stress data-based DCLM and Bayesian probabilistic recursion are described as follows.

For many actual steel bridges, the monitoring extreme stress data is coupled by multiple load effects, and the signal with local trends mainly originates from temperature load effects. Therefore, based on equations (3)-(5), the first-order DCLM is usually enough to predict the coupled extreme stresses and it can be built as follows.

Monitoring equation is

$$
\begin{aligned}
y_{t} & =\theta_{t}+v_{t}, \\
v_{t} & \sim \mathrm{N}\left(0, e^{-0.0004 t^{2}} V\right) \Rightarrow y_{t}=\mathbf{F} \boldsymbol{\theta}_{t}+v_{t}, \\
\mathbf{F} & =(10), \\
\boldsymbol{\theta}_{t} & =\left(\theta_{t}, \beta_{t}\right)^{T} \\
v_{t} & \sim \mathrm{N}\left(0, e^{-0.0004 t^{2}} V\right) .
\end{aligned}
$$

State equation is

$$
\begin{aligned}
\theta_{t} & =\theta_{t-1}+\beta_{t-1}+w_{t, 1}, \\
\beta_{t} & =\beta_{t-1}+w_{t, 2}, \mathbf{w}_{t}=\left(w_{t, 1}, w_{t, 2}\right)^{T} \sim \mathrm{N}\left(0, \mathbf{W}_{t}\right), \\
\Rightarrow \boldsymbol{\theta}_{t} & =\mathbf{G} \boldsymbol{\theta}_{t-1}+\mathbf{w}_{t}, \mathbf{w}_{t} \sim \mathrm{N}\left(0, \mathbf{W}_{t}\right), \\
\boldsymbol{\theta}_{t} & =\left(\theta_{t} \beta_{t}\right)^{T}, \mathbf{w}_{t}=\left(w_{t, 1} w_{t, 2}\right)^{T}, \\
\mathbf{G} & =\left(\begin{array}{cc}
1 & 1 \\
0 & 1
\end{array}\right), \\
\mathbf{W}_{t} & =\left(\begin{array}{cc}
W_{t, 1} & 0 \\
0 & W_{t, 2}
\end{array}\right) .
\end{aligned}
$$

Initial information is

$$
\begin{aligned}
& p\left(\theta_{t-1} \mid \mathbf{D}_{\theta_{t-1}}\right) \approx \sum_{i_{1}=1}^{g_{1}} \alpha_{t-1}^{i_{1}} \mathrm{~N}\left(\theta_{t-1} ; \mu_{t-1}^{i_{1}}, \phi_{t-1}^{i_{1}}\right), \\
& p\left(\beta_{t-1} \mid \mathbf{D}_{\beta_{t-1}}\right) \approx \sum_{i_{2}=1}^{g_{2}} \alpha_{t-1}^{i_{2}} \mathrm{~N}\left(\beta_{t-1} ; \mu_{t-1}^{i_{2}}, \phi_{t-1}^{i_{2}}\right),
\end{aligned}
$$

where $y_{t}$ is the monitoring coupled extreme stress data at time $t ; V$ is approximately estimated with the variance of the differences between the historical states and historical monitoring coupled extreme stresses; $\mathbf{w}_{t}=\left(w_{t, 1}, w_{t, 2}\right)^{T}$ and $v_{t}$ are internally independent and mutually independent; $\theta_{t}$ is the level (or state) of the coupled extreme stress data at time $t$; the initial state information can be approximately obtained with cubical smoothing algorithm with five-point approximation $[2,17]$ through resampling the monitoring coupled extreme stress data at and before time $t ; \beta_{t}$ is the state change at time $t ; \mathbf{W}_{t}$ is the variance matrix about $\mathbf{w}_{t}$.

Initial information in (18) includes two mixture Gaussian PDFs; thus, there are $g 1 \times g 2$ DLMs. Each DLM has the same monitoring equation and state equation and can be, respectively, obtained with the following.

Monitoring equation about the $J^{\text {th }}$ DLM is the same as (16), where $V_{t}$ is substituted by $V_{t, J}$.

State equation about the $J^{\text {th }}$ DLM is the same as (17), where $\mathbf{W}_{t, J}=\left(\begin{array}{cc}W_{t, 1} & 0 \\ 0 & W_{t, 2}\end{array}\right)$.

Initial information about the $J^{\text {th }}$ DLM is

$$
\begin{aligned}
& p\left(\theta_{t-1} \mid \mathbf{D}_{\theta_{t-1}}\right) \approx \mathrm{N}\left(\theta_{t-1} ; \mu_{t-1}^{i_{1}}, \phi_{t-1}^{i_{1}}\right), \quad i_{1} \in\left\{1,2, \ldots, g_{1}\right\}, \\
& p\left(\beta_{t-1} \mid \mathbf{D}_{\beta_{t-1}}\right) \approx \mathrm{N}\left(\beta_{t-1} ; \mu_{t-1}^{i_{2}}, \phi_{t-1}^{i_{2}}\right), \quad i_{2} \in\left\{1,2, \ldots, g_{2}\right\}, \\
& \Rightarrow\left(\boldsymbol{\theta}_{t-1, J} \mid \mathbf{D}_{\boldsymbol{\theta}_{t-1, J}}\right) \sim \mathrm{N}\left(\mathbf{M}_{t-1, J}, \mathbf{C}_{t-1, J}\right), \\
& J \in\left\{1,2, \ldots, \prod_{i=1}^{2} g_{i}\right\}, \\
& \boldsymbol{\theta}_{t-1, J}=\left(\begin{array}{ll}
\theta_{t-1} & \beta_{t-1}
\end{array}\right)_{J}^{T} \\
& \mathbf{D}_{\theta_{t-1, J}}=\left(\begin{array}{ll}
\mathbf{D}_{\theta_{t-1}} & \mathbf{D}_{\beta_{t-1}}
\end{array}\right)_{J}^{T}, \\
& \mathbf{M}_{t-1, J}=\left(\begin{array}{ll}
\mu_{t-1}^{i_{1}} & \mu_{t-1}^{i_{2}}
\end{array}\right)_{J}^{T}, \\
& \mathbf{C}_{t-1, J}=\left(\begin{array}{cc}
\phi_{t-1}^{i_{1}} & 0 \\
0 & \phi_{t-1}^{i_{2}}
\end{array}\right)_{J}
\end{aligned}
$$

where $\mathbf{W}_{t, J}$ can be approximately solved with (7).

Based on equations (3)-(14) and bridge monitoring coupled extreme stress data, Bayesian probabilistic cycle recursive processes of DLM and DCLM (see equations (16)-(19)) can be achieved; furthermore, bridge extreme stress prediction can be made with (13). 


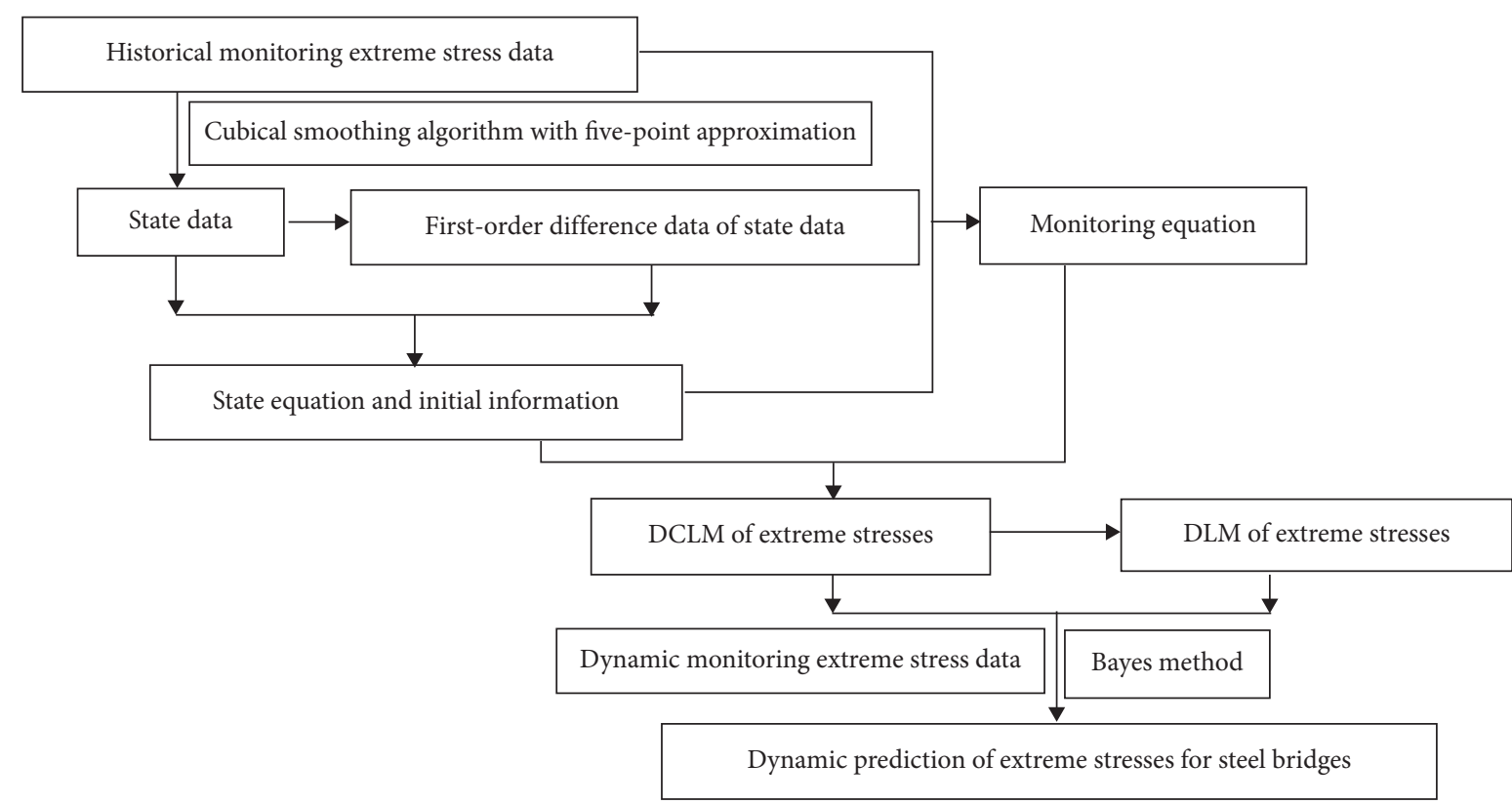

Figure 3: The flowcharts of Section 3.

\section{Monitoring Mechanism of the DCLM}

Model monitoring has three purposes. The first is to identify where model prediction function declines. The second is to cope with the faults and to monitor and update the model. The third is to improve the accuracy of future prediction.

In this paper, the main idea of model monitoring mechanism is to use one or more alternative models to compare and evaluate model performance.

According to the research studies in [1, 34-36], model monitoring is achieved through Bayesian factors under the normal assumption. The main idea is firstly to build an alternative model and then to combine an existing probability model for constructing the formula of Bayesian factors.

In this paper, the adopted probability distribution density function of the alternative model and one-step prediction model are, respectively, in the following equations:

$$
\begin{aligned}
& p_{1}=\left(2 \pi k^{2}\right)^{-0.5} \exp \left(\frac{-0.5 e_{t e}^{2}}{k^{2}}\right), \\
& p_{0}=(2 \pi)^{-0.5} \exp \left(-0.5 e_{t e}^{2}\right),
\end{aligned}
$$

where $e_{t e}=\left(y_{t}-\sum_{J=1}^{\prod_{i=1}^{n} G_{i}}\left(p_{t-1, J} f_{t, J}\right) / \int_{-\infty}^{+\infty}\left(y_{t}-f_{t}\right)^{2} \sum_{J=1}^{\prod_{i=1}^{n} G}\right.$ $\left.p_{t-1, J} \mathrm{~N}\left(f_{t, J}, Q_{t, J}\right) \mathrm{d} y_{t}\right)$ is the standard prediction error obtained with (13) and $k$ is the standard deviation of $e_{t e}$.

The Bayesian factor for $p_{0}(\cdot)$ versus $p_{1}(\cdot)$ based on the observed value of $y_{t}$ is defined as

$$
B(t)=\frac{p_{0}\left(y_{t} \mid \mathbf{D}_{t-1}\right)}{p_{1}\left(y_{t} \mid \mathbf{D}_{t-1}\right)}
$$

where $p_{0}\left(y_{t} \mid \mathbf{D}_{t-1}\right)$ is the one-step predictive PDF of monitoring daily extreme stress data; $p_{1}\left(y_{t} \mid \mathbf{D}_{t-1}\right)$ is PDF of the alternative model, namely, the routine or standard PDF;
$B(t)$ is the Bayesian factor for $p_{0}\left(y_{t} \mid \mathbf{D}_{t-1}\right)$ versus $p_{1}\left(y_{t} \mid \mathbf{D}_{t-1}\right)$ based on the observed value of $y_{t}$.

Furthermore, according to (20)-(22), the Bayesian factors can be obtained with

$$
B(t)=k \exp \left\{-0.5 e_{t e}^{2}\left(1-k^{-2}\right)\right\}
$$

where

$$
e_{t e}=\left(y_{t}-\sum_{i=1}^{n}\left(p_{t-1, i} f_{t, i}\right) / \int_{-\infty}^{+\infty}\left(y_{t}-\right.\right.
$$

$\left.\left.\sum_{i=1}^{n}\left(p_{t-1, i} f_{t, i}\right)\right)^{2} \sum_{i=1}^{n} p_{t-1, i} \mathrm{~N}\left(f_{t, i}, Q_{t, i}\right) \mathrm{d} y_{t}\right)$ is the standard prediction error and $k$ is the standard deviation of $e_{t e}$.

For integers $N=1, \ldots, t$, the Bayesian factor for $p_{0}(\cdot)$ versus $p_{1}(\cdot)$ based on the sequence of $N$ consecutive monitoring daily extreme stress data $y_{t}, y_{t-1}, \ldots, y_{t-N+1}$ is built as (24); namely, the built formula $B_{t}(N)$ of the cumulative Bayesian factor is

$$
B_{t}(N)=\prod_{r=t-N+1}^{t} B_{r}=\frac{p_{0}\left(y_{t}, y_{t-1}, \ldots, y_{t-N+1} \mid \mathbf{D}_{t-N}\right)}{p_{1}\left(y_{t}, y_{t-1}, \ldots, y_{t-N+1} \mid \mathbf{D}_{t-N}\right)}
$$

where $B_{t}(N)$ is the cumulative Bayesian factor, which measures the evidence provided by the recent (up to and including time $t$ ) $N$ consecutive observations $y_{t}, y_{t-1}, \ldots, y_{t-N+1}$.

In this paper, according to the engineering experience, the adopted monitoring criterion $[1,34-36]$ is as follows: if $k=3$ and $B(t)<0.15$, then the corresponding monitoring data is abnormal, which needs to be removed; otherwise, the monitoring data is normal.

After removing the abnormal data, if the cumulative Bayesian factor is bigger, the prediction precision of DCLM is better. The uncertainty of DCLM is smaller. 


\section{Application to an Existing Steel Bridge}

Fumin bridge in Tianjin city of China, taken for the actual example [23, 36-38], is an unsymmetrical single-tower and self-anchored suspension bridge with 3D curved cables, which was designed with three-span arrangements shown in Figure 4 . The total length of this bridge is $340.3 \mathrm{~m}$, and the width is $40 \mathrm{~m}$. The deck is mainly composed of two steel box girders with a short prestressed concrete segment at the right end anchorage, which can be seen in [23, 37, 38]; the monitoring sections of the deck are not affected by the short prestressed concrete segment; the main cables in the main span are anchored to the deck, whereas those in the side span on a vertical plane are anchored to the ground. Most parts of the bridge are made of steel, and the allowable stress of the steel is $345 \mathrm{MPa}$. About this bridge, the girder system is mainly composed of upstream and downstream girders and the crossbeam, and the support conditions are indicated in $[37,38]$. The detailed design information can be obtained in [23, 37, 38].

For the girder system of Fumin bridge, according to the monitoring program [38], there are seven monitoring sections, namely, section $A$, section $B$, section $G$, section $C$, section $\mathrm{D}$, section $\mathrm{E}$, and section $\mathrm{F}$. The positions of these sections are displayed in Figure 5, where sections A, B, and G are in the side span; sections $\mathrm{C}, \mathrm{D}, \mathrm{E}$, and $\mathrm{F}$ are in the main span. These positions play a very important role in controlling the safety-based structural performance. In the measure program for the girder system [38], the sampling rate about the strain data is $2.5 \mathrm{~Hz}$ sampling frequency. The longitudinal hourly monitoring extreme strain data about these seven sections are monitored through an array of Fiber Bragg Grating (FBG) strain sensors, and the corresponding extreme stress data can be obtained with the monitoring extreme strain multiplied by modulus of elasticity.

When the traffic flow magnitude is extremely large, the seven sections are monitored. The dynamic coupled extreme stresses mainly caused by temperature load and vehicle load are nonstationary and can be used to build DCLM.

Among the seven sections, section D is the most dangerous section, so section $\mathrm{D}$ is adopted as the research object. Section D installed three sensors (see Figure 6): FBG01012, FBG01015, and FBG01005, where the monitoring point for FBG01012 is the most dangerous point. Therefore, the hourly coupled extreme stress data of FBG01012 is applied to build DCLM for dynamically predicting hourly coupled extreme stress about section D. The stresses from the dead weight of the steel structure and the concrete deck are not included in the coupled extreme stress data. According to $[23,36]$, it can be known that the stress value caused by the total dead weight is $226 \mathrm{MPa}$. Considering the factor (the value is $1.15[23,36])$ assigned to the data provided by the sensors, the maximum extreme stress value caused by the total live loads is $((345-226) / 1.15)=103.5 \mathrm{MPa}[23]$.

From August 25 to September 5, 2009, the extreme stresses at the installed point of FBG01012 were monitored for 287 hours, and the hourly monitoring coupled extreme stress data collected by sensor FBG01192 is shown in Figure 7.

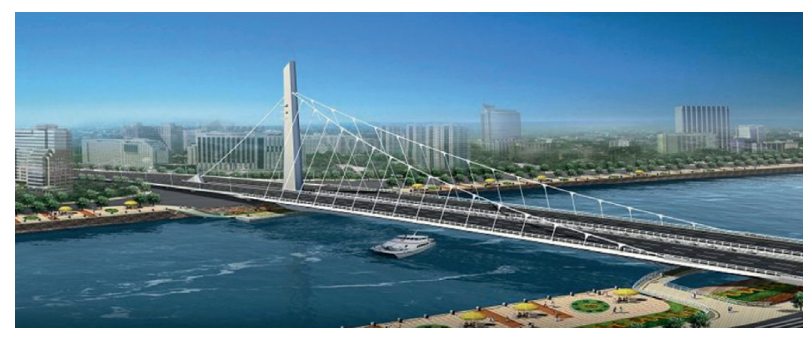

Figure 4: A view of Fumin bridge [23, 36].

Now, to build the DCLM with the monitoring coupled extreme stress data shown in Figure 7, the ADF test based on the $\mathrm{R}$ programming language [30-33] is applied to determine the order of monitoring coupled extreme stress data of the previous 287 hours. The test results show that the firstorder differenced sequence of monitoring coupled extreme stresses is tested to be stationary. Therefore, the local trend order of monitoring coupled data is one; furthermore, Section 3 is reasonable and available for this example.

Based on the monitoring extreme stress data of the previous 287 hours, with the cubical smoothing algorithm with five-point approximation $[2,17,36]$ and the difference method [39], the initial information can be both approximately obtained and shown in Figures 7 and 8 . They can be both simulated with two mixture normal distributions through the least residual error quadratic sum method [2].

Based on (16)-(18) and the monitoring extreme stress data of the previous 287 hours shown in Figure 7, the built DCLM are as follows.

Monitoring equation is

$$
\begin{aligned}
y_{t} & =\theta_{t}+v_{t}, v_{t} \sim \mathrm{N}\left(0,0.016 e^{-0.0004 t^{2}}\right), \\
\Rightarrow y_{t} & =\mathbf{F} \boldsymbol{\theta}_{t}+v_{t}, \\
\mathbf{F} & =\left(\begin{array}{ll}
1 & 0
\end{array}\right), \\
\boldsymbol{\theta}_{t} & =\left(\theta_{t}, \beta_{t}\right)^{T}, \\
v_{t} & \sim \mathrm{N}\left(0,0.016 e^{-0.0004 t^{2}}\right) .
\end{aligned}
$$

State equation is the same as equation (17). The initial information is

$$
\begin{aligned}
p\left(\theta_{t-1} \mid \mathbf{D}_{t-1,1}\right) \approx & 0.6048 \times \mathrm{N}\left(\theta_{t-1} ; 42.6755,0.3012\right) \\
& +0.3952 \times \mathrm{N}\left(\theta_{t-1} ; 44.3965,0.0990\right), \\
p\left(\beta_{t-1} \mid \mathbf{D}_{t-1,2}\right) \approx & 0.1421 \times \mathrm{N}\left(\beta_{t-1} ;-0.0394,0.0003\right) \\
& +0.8579 \times \mathrm{N}\left(\beta_{t-1} ; 0.0071,0.0068\right),
\end{aligned}
$$

where $\mathbf{w}_{t}=\left(w_{t, 1}, w_{t, 2}\right)^{T}$ and $v_{t}$ are internally independent and mutually independent; $\theta_{t}$ is the level (or state) of the extreme stress data at time $t ; \beta_{t}$ is the state change at time $t$.

Based on (17)-(18), (25)-(26), and the monitoring coupled extreme stress data shown in Figure 7 , the four built DLMs are as follows.

(1) The first DLM is as follows. 


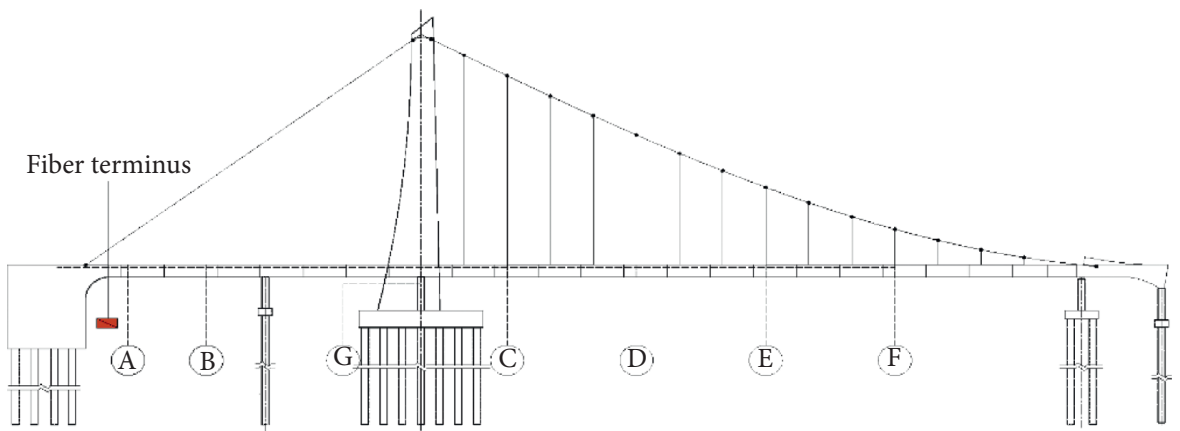

Figure 5: The layout of monitoring sections about Tianjin's Fumin bridge $[23,36,38]$.

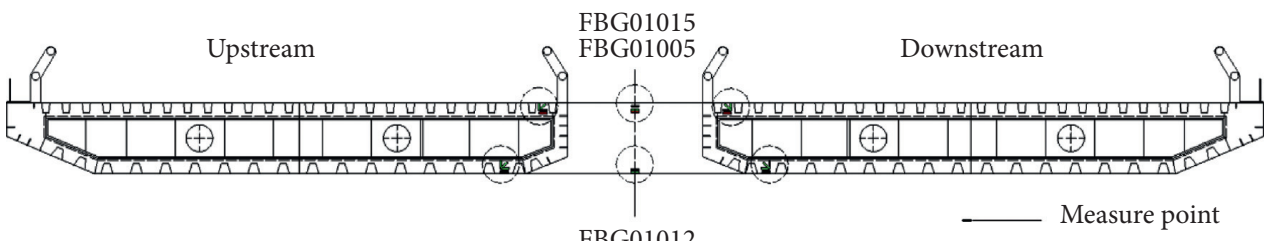

Figure 6: Sensors about section D [23, 36, 38].

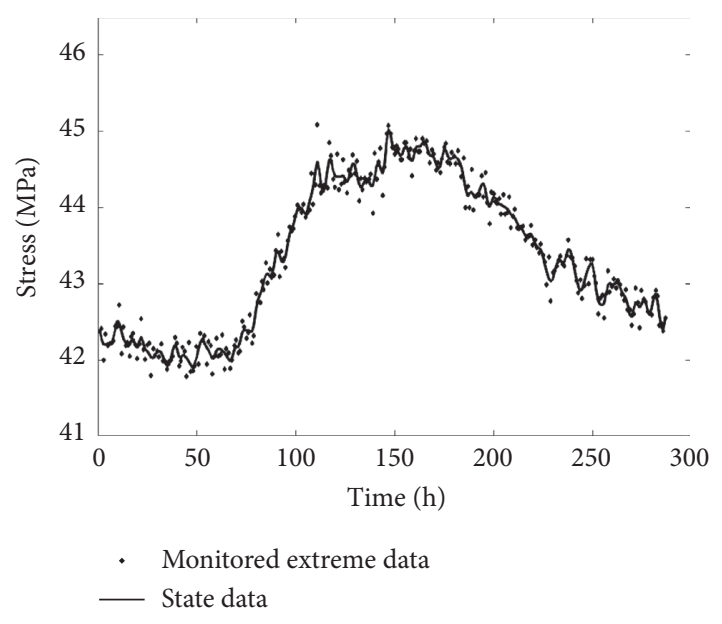

FIgURE 7: Monitoring extreme stress data and state data.

Monitoring equation is the same as equation (25). State equation is

$$
\begin{aligned}
\boldsymbol{\theta}_{t} & =\mathbf{G} \boldsymbol{\theta}_{t-1}+\mathbf{w}_{t}, \\
\mathbf{w}_{t} & \sim \mathrm{N}\left(0, W_{t}\right), \\
\boldsymbol{\theta}_{t} & =\left(\begin{array}{ll}
\theta_{t} & \beta_{t}
\end{array}\right)^{T}, \\
\mathbf{w}_{t} & =\left(\begin{array}{ll}
w_{t, 1} & w_{t, 2}
\end{array}\right)^{T}, \\
\mathbf{G} & =\left(\begin{array}{cc}
1 & 1 \\
0 & 1
\end{array}\right), \\
\mathbf{W}_{t} & =-\mathbf{G C}_{t-1} \mathbf{G}^{T}+\frac{\mathbf{C}_{t-1}}{0.8} .
\end{aligned}
$$

The initial information is

$$
\begin{aligned}
p\left(\theta_{t-1} \mid \mathbf{D}_{t-1,1}\right) & \approx \mathrm{N}\left(\theta_{t-1} ; 42.6755,0.3012\right) \\
p\left(\beta_{t-1} \mid \mathbf{D}_{t-1,2}\right) & \approx \mathrm{N}\left(\beta_{t-1} ;-0.0394,0.0003\right) \\
\Rightarrow \mathbf{C}_{t-1} & =\left(\begin{array}{cc}
0.3012 & 0 \\
0 & 0.0003
\end{array}\right) .
\end{aligned}
$$

(2) The second DLM is as follows.

Monitoring equation is the same as equation (25). State equation is the same as equation (27). The initial information is

$$
\begin{aligned}
p\left(\theta_{t-1} \mid \mathbf{D}_{t-1,1}\right) & \approx \mathrm{N}\left(\theta_{t-1} ; 42.6755,0.3012\right) \\
p\left(\beta_{t-1} \mid \mathbf{D}_{t-1,2}\right) & \approx \mathrm{N}\left(\beta_{t-1} ; 0.0071,0.0068\right) \\
\Rightarrow C_{t-1} & =\left(\begin{array}{cc}
0.3012 & 0 \\
0 & 0.0068
\end{array}\right) .
\end{aligned}
$$

(3) The third DLM is as follows.

Monitoring equation is the same as equation (25). State equation is the same as equation (27). The initial information is

$$
\begin{aligned}
p\left(\theta_{t-1} \mid \mathbf{D}_{t-1,1}\right) & \approx \mathrm{N}\left(\theta_{t-1} ; 44.3965,0.0990\right), \\
p\left(\beta_{t-1} \mid \mathbf{D}_{t-1,2}\right) & \approx \mathrm{N}\left(\beta_{t-1} ;-0.0394,0.0003\right), \\
\Rightarrow C_{t-1} & =\left(\begin{array}{cc}
0.0990 & 0 \\
0 & 0.0003
\end{array}\right) .
\end{aligned}
$$

(4) The fourth DLM is as follows.

Monitoring equation is the same as equation (25). State equation is the same as equation (27). 


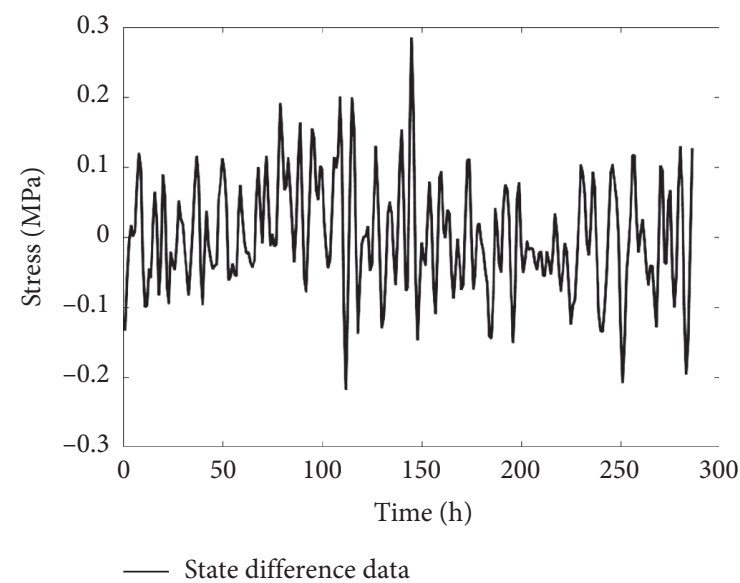

Figure 8: State change data (or state difference data).

The initial information is

$$
\begin{aligned}
p\left(\theta_{t-1} \mid \mathbf{D}_{t-1,1}\right) & \approx \mathrm{N}\left(\theta_{t-1} ; 44.3965,0.0990\right), \\
p\left(\beta_{t-1} \mid \mathbf{D}_{t-1,2}\right) & \approx \mathrm{N}\left(\beta_{t-1} ; 0.0071,0.0068\right), \\
\Rightarrow C_{t-1} & =\left(\begin{array}{cc}
0.0990 & 0 \\
0 & 0.0068
\end{array}\right) .
\end{aligned}
$$

Based on the monitoring coupled extreme stress data from the $287^{\text {th }}$ hour to the $1149^{\text {th }}$ hour, the predicted extreme stresses and prediction precision from the $288^{\text {th }}$ hour to the $1150^{\text {th }}$ hour are, respectively, solved with (8)-(14), (17), and (25)-(31); the results are shown in Figures 9 and 10. From Figure 9, it can be seen that predicted coupled extreme stresses well fit the changing rules of monitoring coupled extreme stress data. Furthermore, with the distant updating of monitoring coupled extreme stress data, the prediction precision gradually increases as shown in Figure 10, which signifies that it is reasonable to predict the structural dynamic coupled extreme stresses with the built DCLM. The predicted coupled extreme stresses are all smaller than 103.5 MPa, so section D is safe.

In the prediction processes, the monitoring mechanism is adopted, and the single Bayesian factors are all less than 0.15 , so the monitoring extreme stresses are all normal. The cumulative Bayesian factors increase, so the prediction precision of DCLM is better, and the uncertainty of DCLM is smaller.

ARIMA [30-33, 40], BDLM [36], BFDLM [23], and Fourier regressive functions [23] are utilized for fair comparison with the proposed DCLM for the prediction effects of bridge coupled extreme stresses, and the predicted results are shown in Figure 11. The mean square errors (MSE) (see equations (15) and (32)) for the predicted extreme stresses of DCLM, ARIMA, BDLM, BFDLM, and Fourier regressive function are, respectively, $0.048,0.1035,0.0953,0.0538$, and 0.4775 . ARIMA method is suitable for short-term prediction; BDLM does not have steady prediction precision; the state equation of BFDLM is complex and relies on very subjective Fourier regressive functions; so the DCLM prediction method is more

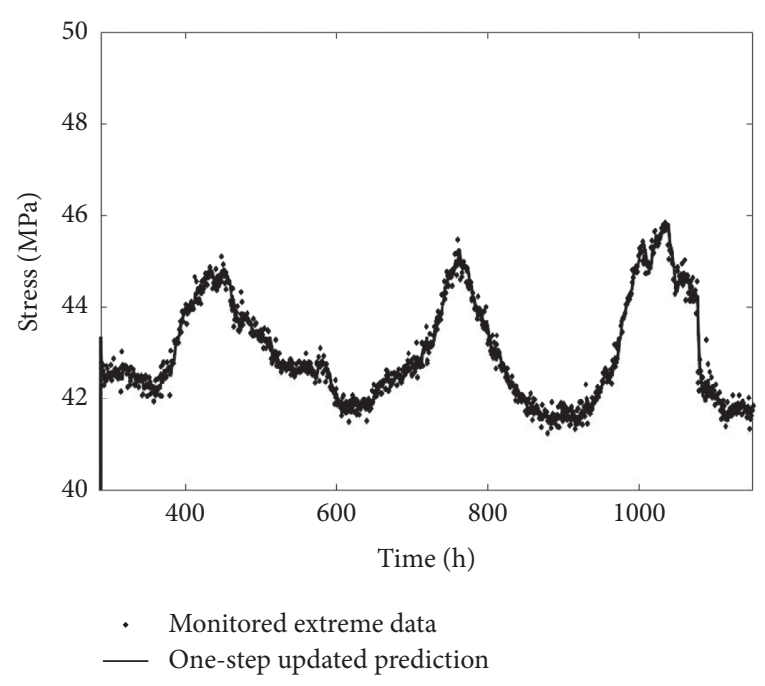

Figure 9: Monitoring stresses and one-step predicted stresses.

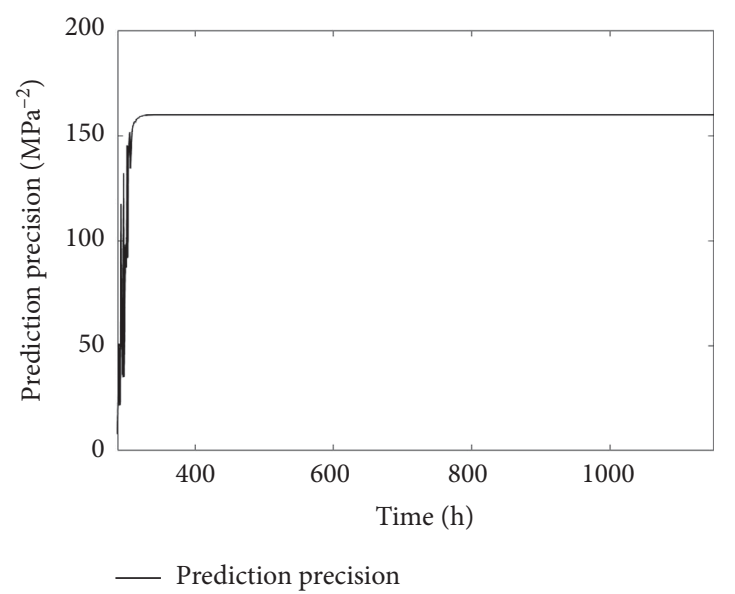

Figure 10: One-step prediction precision.

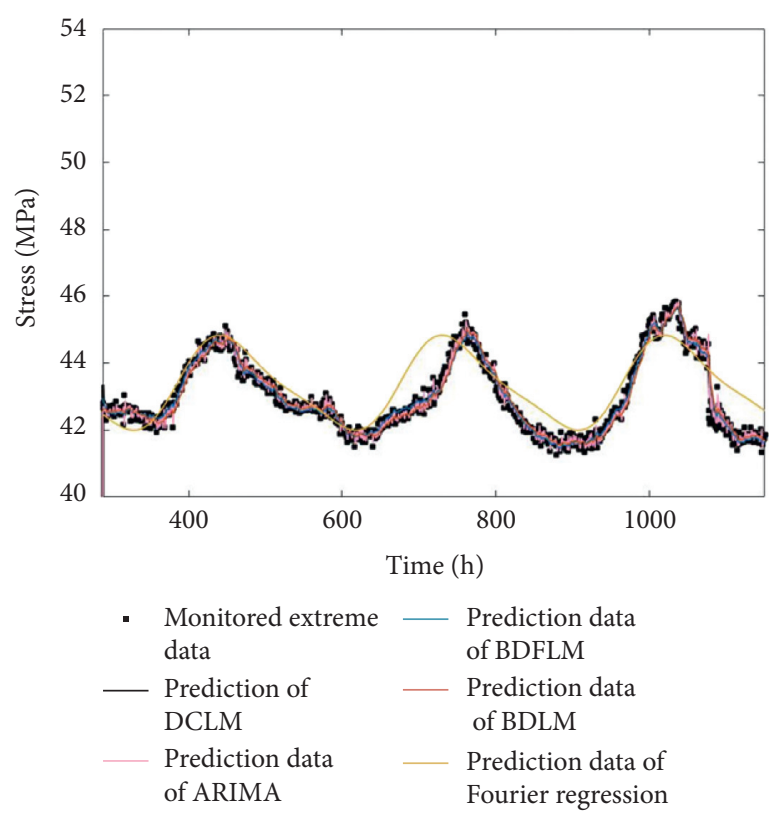

FIGURE 11: Predicted results of the five methods. 


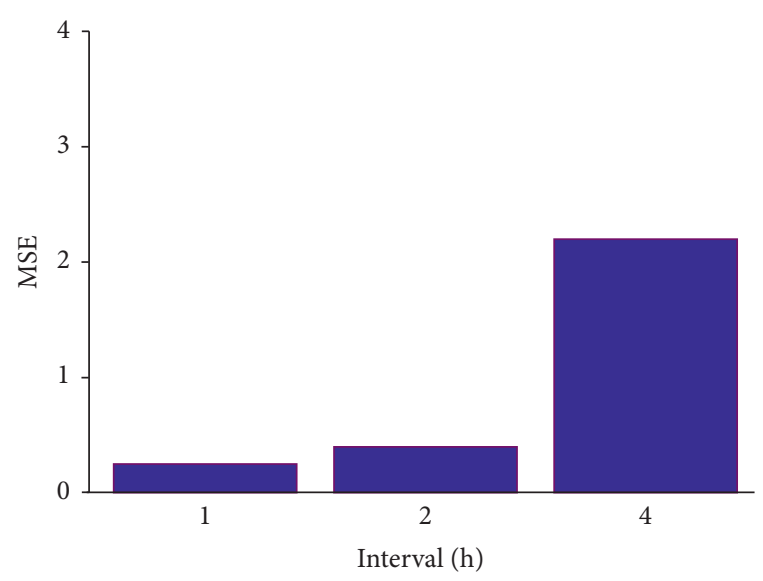

FIGURE 12: MSEs of DCLM about three kinds of time intervals.

effective than the other four methods for predicting coupled extreme stresses.

In this example, the built DCLM has a first-order differential approximation of local polynomials for monitoring coupled extreme stress data at each time; therefore, it can make MSE the smallest among the above five models. MSE is smaller, and the corresponding model is more reasonable, so it can be noticed that the proposed DCLM algorithm is more suitable than the other four models for coupled extreme stress prediction of steel bridges. What is more, the DCLM can be easily and widely used in extreme stress prediction; in particular, the state equation is more feasible than the other four methods.

$$
f_{\mathrm{MSE}}=\sqrt{\sum_{t=288}^{1150} \frac{\left(f_{t}-y_{t}\right)^{2}}{863}}
$$

where $f_{\mathrm{MSE}}$ is the formula of MSE; $f_{t}$ is predicted with (13); $y_{t}$ is the monitoring coupled extreme stress data at time $t$.

According to the description in Section 2.4.2, the extreme stresses with sampling time interval of every-twohours and every-four-hours are predicted, and their MSEs calculated with (15) are shown in Figure 12. It can be noticed from Figure 12 that MSEs increase as the time interval increases. So, the adopted time interval is one hour in this paper.

\section{Conclusions}

This paper first presents the Bayesian dynamic coupled prediction method for coupled extreme stresses of steel bridges based on DCLM and Bayes method, which could effectively and simply deal with the influences of coupling on bridge monitoring information. This method is mainly applied for the monitoring coupled data. In addition, the proposed DCLM has the advantages of simplicity and convenience when dealing with monitoring coupled data; namely, the highly adaptive state equation instead of the complex state curves fitted with the historical monitoring data is reasonably applied in the filtering recursion processes of DCLM based on Bayes method. The proposed method can provide the foundations for bridge reliability prediction and assessment.

\section{Data Availability}

The data used to support the findings of this study are available from the corresponding author upon request.

\section{Conflicts of Interest}

The authors declare no potential conflicts of interest with respect to the research, authorship, and/or publication of this article.

\section{Acknowledgments}

The authors acknowledge the financial support for the research from National Key R\&D Program of China (Grant no. 2019YFC1511005), Fundamental Research Funds for the Central Universities (Grant no. lzujbky-2020-55), and the National Natural Science Foundation of China (Grant no. 51608243).

\section{References}

[1] Y. F. Liu and X. P. Fan, "Bayesian prediction of bridge extreme stresses based on DLTM and monitoring coupled data," Structural Health Monitoring-An International Journal, vol. 19, no. 2, pp. 454-456, 2020.

[2] X. Fan and Y. Liu, "Use of monitored daily extreme stress data for performance prediction of steel bridges: dynamic linear models and Gaussian mixed particle filter," Mechanical Systems and Signal Processing, vol. 121, pp. 841-855, 2019.

[3] Y. F. Liu, D. G. Lu, and X. P. Fan, "Reliability updating and prediction of bridge structures based on proof loads and monitored data," Construction and Building Materials, vol. 66, no. 5, pp. 795-804, 2014.

[4] P. C. Chang, A. Flatau, and S. C. Liu, "Review paper: health monitoring of civil infrastructure," Structural Health Monitoring: An International Journal, vol. 2, no. 3, pp. 257-267, 2003.

[5] T. H. T. Chan, L. Yu, H. Y. Tam et al., "Fiber Bragg grating sensors for structural health monitoring of Tsing Ma bridge: background and experimental observation," Engineering Structures, vol. 28, no. 5, pp. 648-659, 2006.

[6] J. M. Ko and Y. Q. Ni, "Technology developments in structural health monitoring of large-scale bridges," Engineering Structures, vol. 27, no. 12, pp. 1715-1725, 2005.

[7] I. Hodgson, "Personal discussion for the acquisition of the real data from the monitoring of the I-39 northbound bridge over the Wisconsin river," Lehigh University, Bethlehem, PA, USA, 2007.

[8] J. Zhou, X. Li, R. Xia, J. Yang, and H. Zhang, "Health monitoring and evaluation of long-span bridges based on sensing and data analysis: a survey," Sensors, vol. 17, no. 3, p. 603, 2017.

[9] H. M. Lee and H. S. Park, "Measurement of maximum strain of steel beam structures based on average strains from vibrating wire strain gages," Experimental Techniques, vol. 37, no. 2, pp. 23-29, 2013.

[10] C. Bedon, E. Bergamo, M. Izzi, and S. Noè, "Prototyping and validation of MEMS accelerometers for structural health monitoring - the case study of the Pietratagliata cable-stayed 
bridge," Journal of Sensor and Actuator Networks, vol. 7, no. 3, pp. 1-18, 2018.

[11] K.-Y. Wong, "Instrumentation and health monitoring of cable-supported bridges," Structural Control and Health Monitoring, vol. 11, no. 2, pp. 91-124, 2004.

[12] Z. Chen, Y. Bao, H. Li, and B. F. Spencer, "LQD-RKHS-based distribution-to-distribution regression methodology for restoring the probability distributions of missing SHM data," Mechanical Systems and Signal Processing, vol. 121, pp. 655674, 2019.

[13] M. Silva, A. Santos, E. Figueiredo, R. Santos, C. Sales, and J. C. W. A. Costa, "A novel unsupervised approach based on a genetic algorithm for structural damage detection in bridges," Engineering Applications of Artificial Intelligence, vol. 52, pp. 168-180, 2016.

[14] C. Bedon, M. Dilena, and A. Morassi, "Ambient vibration testing and structural identification of a cable-stayed bridge," Meccanica, vol. 51, no. 11, pp. 2777-2796, 2016.

[15] O. Abdeljaber, O. Avci, S. Kiranyaz, M. Gabbouj, and D. J. Inman, "Real-time vibration-based structural damage detection using one-dimensional convolutional neural networks," Journal of Sound and Vibration, vol. 388, pp. 154-170, 2017.

[16] C.-W. Kim, Y. Zhang, Z. Wang, Y. Oshima, and T. Morita, "Long-term bridge health monitoring and performance assessment based on a Bayesian approach," Structure and Infrastructure Engineering, vol. 14, no. 7, pp. 883-894, 2018.

[17] X. P. Fan and Y. F. Liu, "New dynamic prediction approach for the reliability indexes of bridge members based on SHM data," ASCE Journal of Bridge Engineering, vol. 23, no. 12, Article ID 06018004, 2018.

[18] J. Wang and X. Liu, "Evaluation and Bayesian dynamic prediction of deterioration of structural performance," Structure and Infrastructure Engineering, vol. 6, no. 6, pp. 663-674, 2010.

[19] D. M. Frangopol, A. Strauss, and S. Kim, "Use of monitoring extreme data for the performance prediction of structures: general approach,” Engineering Structures, vol. 30, no. 12, pp. 3644-3653, 2008.

[20] Y. Q. Ni, X. G. Hua, and J. M. Ko, "Reliability-based assessment of bridges using long-term monitoring data," Key Engineering Materials, vol. 321-323, pp. 217-222, 2006.

[21] F. N. Catbas, M. Susoy, and D. M. Frangopol, "Structural health monitoring and reliability estimation: long span truss bridge application with environmental monitoring data," Engineering Structures, vol. 30, no. 9, pp. 2347-2359, 2008.

[22] D. M. Frangopol, A. Strauss, and S. Kim, "Bridge reliability assessment based on monitoring," Journal of Bridge Engineering, vol. 13, no. 3, pp. 258-270, 2008.

[23] Y. Liu and X. Fan, "A data assimilation method about Bayesian Fourier dynamic linear prediction of periodic extreme stresses for steel bridges," Mechanical Systems and Signal Processing, vol. 128, pp. 82-92, 2019.

[24] Y. Chen and P. L. Durango-Cohen, "Development and field application of a multivariate statistical process control framework for health-monitoring of transportation infrastructure," Transportation Research Part B: Methodological, vol. 81, pp. 78-102, 2015.

[25] C. Cheny and J.-M. Kauffmann, "Information losses in decoupling space harmonics effects for an induction drive," Mathematics and Computers in Simulation, vol. 46, no. 3-4, pp. 361-372, 1998.
[26] X. J. Guo, "Structural damage identification based on the particle filter and reliability analysis," Southeast University, Nanjing China, 2016.

[27] R. Kromanis and P. Kripakaran, "Data-driven approaches for measurement interpretation: analysing integrated thermal and vehicular response in bridge structural health monitoring," Advanced Engineering Informatics, vol. 34, pp. 46-59, 2017.

[28] R. Kromanis and P. Kripakaran, "Predicting thermal response of bridges using regression models derived from measurement histories," Computers \& Structures, vol. 136, pp. 64-77, 2014.

[29] R. Kromanis and P. Kripakaran, "Support vector regression for anomaly detection from measurement histories," Advanced Engineering Informatics, vol. 27, no. 4, pp. 486-495, 2013.

[30] R. H. Shumway and D. S. Stoffer, Time Series Analysis and Its Applications with $R$ Examples, Springer, Berlin, Germany, 2010.

[31] P. S. P. Cowpertwait and A. V. Metcalfe, Introductory Time Series with R, Springer series, Berlin, Germany, 2009.

[32] D. A. Dickey and W. A. Fuller, "Distribution of the estimators for autoregressive time series with a unit root," Journal of the American Statistical Association, vol. 74, no. 366a, pp. 427431, 1979.

[33] R. H. Shumway and D. S. Stoffer, Time Series and Its Applications with $R$ Examples, Springer, Berlin, Germany, 4th edition, 2017.

[34] M. West and J. Harrison, Bayesian Forecasting and Dynamic Models, Springer, Berlin, Germany, 2nd edition, 1997.

[35] G. Petris, S. Petrone, and P. Campagnoli, Dynamic Linear Models with R, Springer, Berlin, Germany, 2009.

[36] X. P. Fan, Reliability updating and Bayesian prediction of bridges based on proof loads and monitored data, Ph.D. thesis, Harbin Institute of Technology, Harbin, China, 2014.

[37] Y. Sun, H.-P. Zhu, and D. Xu, "A specific rod model based efficient analysis and design of hanger installation for selfanchored suspension bridges with 3D curved cables," Engineering Structures, vol. 110, pp. 184-208, 2016.

[38] S. F. Chen and D. W. Yang, As-built drawings and measurement programs of Fumin bridge health monitoring project, Ph.D. thesis, Harbin Institute of Technology, Harbin, China, 2010.

[39] H. Massaldi and J. A. Maymó, "Error in handling finite conversion reactor data by the differential method," Journal of Catalysis, vol. 14, no. 1, pp. 61-68, 1969.

[40] Z. Zhao, "Health monitoring data modeling and reliability analysis for Yitong river bridge based on ARMA model," M.S. thesis, Harbin: School of Civil Engineering, Harbin Institute of Technology, Harbin, China, 2012. 